Nicolas Patin

\title{
Von den Schützengräben zur NSDAP. Kriegskultur und Politisierung der nationalsozialistischen Reichstagsabgeordneten
}

Seit dem grundlegenden Werk von George L. Mosse hat der Leitbegriff der »Kriegskultur « zu wertvollen Erkenntnissen über das frühe 20. Jahrhundert geführt ${ }^{1}$. Auf der Grundlage der Analyse, die der amerikanische Historiker über den »Mythos « des Ersten Weltkrieges und dessen Auswirkungen auf die Nachkriegsgesellschaften publizierte, wurden inzwischen zahlreiche Studien veröffentlicht. Zwei Jahrzehnte später erscheinen Mosses Gedanken immer noch aktuell. Dabei wurde manchmal vergessen, dass sein Werk eine Art Essay war, der auf einer Geschichte der Repräsentation und der Sprache aufbaute; die Sozialgeschichte - und erst recht die Politikgeschichte - bildeten dabei nur den großen Interpretationsrahmen. Die erste große Erkenntnis in Mosses Werk, die Vorstellung einer Banalisierung bzw. Trivialisierung des Krieges, der Gewalt und des Todes wurde von den darauf aufbauenden historischen Forschungen weitgehend bestätigt ${ }^{2}$.

Mosses zweite These einer generellen Brutalisierung der politischen Kultur in Deutschland stieß jedoch auf deutliche Kritik ${ }^{3}$. Tatsächlich war dieses Konzept, demzufolge »die Beibehaltung von militanten Einstellungen über den Kriege hinaus « ${ }^{4}$ erfolgte, zu pauschal gehalten. Schon die sozialgeschichtlichen Untersuchungen Richard Bessels aus den 1980er Jahren hatten auf den allenfalls mittelbaren Zusammenhang zwischen den Formen der Gewalt im Krieg und in der Weimarer Republik hingewiesen ${ }^{5}$. Andere Verfasser bemühten sich aufzuzeigen, dass die Brutalisierung eher als ein langfristiges Phänomen zu deuten sei. Dabei habe der Krieg zwar als Katalysator gewirkt, allerdings weniger entscheidend, als man das auf den ersten Blick annehmen könne ${ }^{6}$. Die kritischen Auseinandersetzungen mit dem Werk Mosses kamen vor allem aus Deutschland selbst, wobei man dort zumeist immer wieder deutlich machen wollte, welche Bedeutung der Generationenanalyse für das Verständnis der Gewalt zukam. Gleichwohl gilt das Konzept der »Kriegskultur « heute im Prinzip als gefestigt, zumal sich darüber gut die Zusammenhänge zwischen den Jahren 1914-1918 und 1939-1945 aufzeigen lassen.

Es brauchte eine ganze Weile, bis diese Hypothesen auch in dem bereits sehr intensiv bearbeiteten Forschungsfeld zum Nationalsozialismus aufgegriffen wur-

1 George L. Mosse, Gefallen für das Vaterland. Nationales Heldentum und namenloses Sterben, Stuttgart 1993. Übersetzung des vorliegenden Aufsatzes durch Bundessprachenamt und Markus Pöhlmann.

2 Ebd., Kapitel 7: Der Prozess der Trivialisierung.

3 Ebd. Vgl. das gleichnamige Kapitel, S. 195-222. Dazu Antoine Prost, Les limites de la brutalisation. In: Vingtième Siècle. Revue d'histoire, 81 (2004), S. 5-20.

4 Mosse, Gefallen für das Vaterland (wie Anm. 1), S. 195.

5 Richard Bessel, Germany after the First World War, Oxford 1993, und Richard Bessel, Political Violence and the Rise of Nazism, The Storm Troopers in Eastern Germany 1925-1934, New Haven, CT, London 1984.

6 Bernd Weisbrod, Violence et culture politique en Allemagne entre les deux guerres. In: Vingtième Siècle. Revue d'histoire, 24 (1992), 1, S. 113-125. 
den: Hat der Erste Weltkrieg die Bildung der nationalsozialistischen Partei stark beeinflusst? Mosse selbst hatte bereits auf Wege zur Beantwortung dieser Frage hingewiesen: Für ihn hatte die »Brutalisierung der Politik « die »politische Rechte während der Weimarer Republik beseelt « ${ }^{7}$. Diese Vorstellung präzisierte er wie folgt: »Der Krieg hatte die Kräfte, die er freisetzte, nicht selbst erzeugt; er gab ihnen nur eine neue Richtung und Dynamik und verhalf ihnen zum Sieg ${ }^{8}$." Das Bild des Gefreiten Hitler, die offensichtliche Militarisierung der Sturmabteilungen (SA), die Uniformen, die Frontsoldatenrhetorik, all dies wies eindeutig darauf hin, dass die Nationalsozialistische Deutsche Arbeiterpartei (NSDAP) im Ersten Weltkrieg, in seiner Gewalt und seiner Sprache wurzelte. Die jüngsten prosopografischen Untersuchungen zum Nationalsozialismus verweisen alle auf diese Matrix des Ersten Weltkrieges. So beginnt das Buch von Christian Ingrao, in dem dieser die Biografien von 80 SS-Intellektuellen analysiert, die zwischen 1941 und 1945 Kriegsverbrechen an der Ostfront begingen, mit dem Satz: »Die erste gemeinsame Erfahrung jener Menschen, die uns hier interessieren, war der Erste Weltkrieg ${ }^{9}$ « Weil der Krieg und die Niederlage von 1918 bei diesen Männern »eine eschatologische Angst $«{ }^{10}$ vor dem Untergang Deutschlands ausgelöst hatten, konnten sie noch zwanzig Jahre später gnadenlos gegen Feinde kämpfen, da sie sie für gefährliche Feinde ihres Vaterland hielten. Der Gedankengang liegt auf der Hand: Aus der bereits vor 1914 perzipierten Angst vor der »Einkreisung " ${ }^{11}$ entwickelte sich in Deutschland der Mythos vom »Verteidigungskrieg « ${ }^{12}$ gegen eine »Welt von Feinden ${ }^{13}$. Daher die beständige Angst; daher die noch zwanzig Jahre später virulente Vorstellung von der Notwendigkeit, die potenziellen Feinde vollständig vernichten zu müssen und die Ängste in eine »imperiale Hoffnung $«{ }^{14}$ umzuwandeln.

Grundsätzlich wird man diese Interpretation nicht in Frage stellen. Aber - und darum geht es in diesem Beitrag - wenn man sich für die Politisierung der Mitglieder der NSDAP interessiert, dann erscheint der Krieg zwar als ein zentraler Faktor, der jedoch mit anderen, ebenso zentralen Faktoren in Verbindung gebracht werden muss. Michael Wildt beispielsweise unterstreicht in seiner Prosografie der Angehörigen des Reichssicherheitshauptamtes (RSHA) die Bedeutung des Traumas der Inflation von $1923^{15}$. Das ist eine lohnende Spur, denn die entscheidende Bedeutung der sozioökonomischen Lage im damaligen Deutschland gerät oft in Ver-

\footnotetext{
Mosse, Gefallen für das Vaterland (wie Anm. 1), S. 222.

Ebd., S. 221.

Christian Ingrao, Hitlers Elite. Die Wegbereiter der nationalsozialistischen Massenmorde, Berlin 2012, S. 15 (frz. Ausg. 2010). Eine umfassende Analyse dieses Werkes findet sich in der Rezension von Nicolas Patin, Comment peut-on être nazi. In: Nonfiction.fr, 26.11.2010.

10 Ingrao, Hitlers Elite (wie Anm. 9), S. 11, 95 und S. 106.

11 Jean-Jacques Becker et Gerd Krumeich, La Grande Guerre. Une histoire franco-allemande, Paris 2008, S. 34.

12 Dieser Begriff erschien regelmäßig im Wortschatz der Rechten und der extremen Rechten. Vgl. z.B. die Rede von Ernst Graf zu Reventlow im Reichstag am 30.11.1929. In: Verhandlungen des Reichstags, IV. Wahlperiode 1928, Bd 426, Berlin 1928, S. 3342 f.

13 Mit diesem Begriff wurde ausgedrückt, dass Deutschland gegen eine unverhältnismäßig große Zahl von Feinden Krieg geführt hatte. Der Abgeordnete Ludwig Haas verwendete diesen Ausdruck zum Beispiel am Rednerpult des Reichstags. Verhandlungen des Reichstags, I. Wahlperiode 1920, Bd 344, Berlin 1920, S. 442.

14 Ingrao, Hitlers Elite (wie Anm. 9), S. 369.

15 Michael Wildt, Generation des Unbedingten. Das Führungskorps des Reichssicherheitshauptamtes, Hamburg 2003, S. 25.
} 
gessenheit ${ }^{16}$. Wenn man die Politisierung der Abgeordneten der NSDAP betrachtet, werden sich weitere Faktoren erschließen.

Weimar war die erste deutsche Republik. Der Reichstag bestand zwar bereits seit der Gründung des Reiches im Jahr 1871. Aber erst die Erweiterung des Wahlrechts auf die Frauen und vor allem die neuen, durch die Weimarer Verfassung von 1919 verliehenen Zuständigkeiten brachten den Reichstag ins Zentrum des politischen Systems. Der NSDAP gehörten 1928 nur zwölf Reichstagsabgeordnete $(2,6 \%)$ an, was nicht einmal zur Bildung einer Fraktion ausreichte. 1930 zogen 107 Nationalsozialisten, und zwar in SA-Uniform, in den Reichstag ein. Im Juli 1932 waren es 230, und nach den Wahlen im November desselben Jahres 196. Daher beteiligten sich von 1928 bis 1932 insgesamt 287 nationalsozialistische Reichstagsabgeordnete an der Zerstörung des parlamentarischen Systems ${ }^{17}$. Die Fragen, die im Folgenden geklärt werden sollen, sind: Welchen Platz hatte der Krieg in der politischen Vorstellungswelt und nach welcher Logik verlief ihre Politisierung? Anhand des hier untersuchten Quellenkorpus wird aufgezeigt werden, dass die Erfahrung des Schützengrabens zwar sehr verbreitet war, dass jedoch weitere Faktoren hinzukamen, die für das weitere Verständnis des Werdegangs dieser Parteifunktionäre eine wesentliche Rolle spielten. Die Untersuchung wird sich auf drei dieser Faktoren konzentrieren: das Alter, den Beruf des Vaters und die landsmannschaftliche Herkunft.

\section{Frontgeneration}

1930 zählten die nationalsozialistische Reichstagsfraktion 80 Prozent, die beiden Fraktionen von 1932 (Juli bzw. November) 75 Prozent Weltkriegsveteranen. Dieser Anteil lag bei mehr als 15 Prozentpunkten über dem Durchschnitt der Reichstagsabgeordneten insgesamt. Aber repräsentierte die NSDAP damit als einzige Partei die Kriegserfahrung? Keineswegs.

Vor 1930 erhoben zwei Parteien Anspruch auf dieses Merkmal: Unter den Reichstagsabgeordneten der Deutschnationalen Volkspartei (DNVP), die dem konservativen Wehrverband "Stahlhelm. Bund der Frontsoldaten « nahestand, befanden sich in den Jahren 1924-1930 zwischen 55 und 65 Prozent Veteranen. Der Reichstagsfraktion der Kommunistischen Partei Deutschlands (KPD) gehörten 1924 bis zu 60 Prozent Veteranen an, bis die personalpolitische Neuausrichtung der Partei vor allem Mandatsträger in den Reichstag brachte, die den Weltkrieg nicht mehr

16 Eine neuere Studie befasst sich mit den Selbstmorden während der Weimarer Republik. Die Selbstmordrate war während des Krieges niedrig und stieg in den Jahren 1923-25 und 1929-32 stark an. Vgl. Christian Goeschel, Suicide in Nazi Germany, Oxford, New York 2009, S. 12-15 und S. 209-212.

17 Alle in diesem Artikel genannten Zahlen stammen aus der Datenbank BIORAB (www. zhsf.uni-koeln.de/biorab/). Sie wurden in einer archivarischen Arbeit in den verschiedenen deutschen Zentren miteinander abgeglichen. Einzelheiten dazu finden sich in meiner Doktorarbeit: Nicolas Patin, La Guerre au Reichstag. Expériences de guerre et imaginaires politiques des députés sous la République de Weimar, 1914-1933, die ich am 27.11.2010 an der Université Paris Ouest Nanterre La Défense verteidigt habe. Dazu Nicolas Patin, La catastrophe allemande. 1674 destins parlementaires, Paris 2014. 
als Soldaten kennengelernt hatten. Mit Ausnahme der Deutschen Demokratischen Partei konnten sämtliche Reichstagsfraktionen zu jedem Zeitpunkt der Weimarer Republik unter ihren Mitgliedern mindestens 50 Prozent Veteranen nachweisen. Das heißt, dass die Kriegserfahrung unter den Abgeordneten insgesamt weit verbreitet war - und bei turbulenten Debatten über die Rechtfertigung des Kampfes von allen Parteien auch lautstark für sich beansprucht wurde.

Die Besonderheit der nationalsozialistischen Fraktion bestand also nicht darin, dass ihre Abgeordneten am Krieg teilgenommen hatten. Sie bestand vielmehr in deren Alter. Das Durchschnittsalter der Reichstagsabgeordneten zum Zeitpunkt ihrer Wahl 1928-1932 lag bei 47 Jahren. Von diesem traditionellen Karriereverlauf gab es eigentlich kaum Abweichungen in den einzelnen Parteien - mit zwei Ausnahmen: Der Altersunterschied der Mitglieder der kommunistischen Fraktion zu denen der konservativen Rechten (51 Jahre) betrug vierzehn Jahre, denn die KPDAbgeordneten zogen mit durchschnittlich 37 Jahren in den Reichstag ein. Ihnen gegenüber saß im Plenarsaal eine nationalsozialistische Fraktion, deren Mitglieder 39 Jahre alt waren. In den Parteien der Mitte waren einige Abgeordnete zu alt, um am Krieg teilgenommen zu haben, bei der KPD und der NSDAP waren sie dagegen zu jung. 80 Prozent der Abgeordneten der Braunhemden gehörten zwar der »Frontgeneration « an, aber die restlichen 20 Prozent waren Teil der für die Front »überflüssige[n] Generation ${ }^{18}$, also jener jungen Männer, die zwar oft in die Armee eingetreten, aber nicht mehr an die Front gekommen waren. Der bekannteste Vertreter dieser Generation war sicherlich Heinrich Himmler ${ }^{19}$. Dieser Altersunterschied findet sich auch zu anderen Fraktionen. So waren die Mitglieder der Sozialdemokratischen Partei Deutschlands (SPD) 1930 durchschnittlich 46 Jahre alt, die der NSDAP aber nur $30^{20}$.

Aufgrund dieses generationellen Aspektes kann der Krieg nicht als einziger Katalysator der Politisierung betrachtet werden. Dabei wäre diese Interpretation verlockend, denn während 86 Prozent der künftigen Abgeordneten der SPD und der KPD bereits vor dem Krieg politisiert waren, war nur eine Handvoll der nationalsozialistischen Abgeordneten vor dem Krieg politisch aktiv gewesen. Der Krieg ließ zwar einen Teil der vor dem Krieg der Arbeiterklasse zugehörigen aktiven Parteimitglieder radikal nach links rücken. Er scheint aber zugleich auf der extremen Rechten eine politische Generation ins Leben gerufen zu haben. Dies war zumindest eine der Schlussfolgerungen, zu denen ich in einem früheren Aufsatz gekommen bin $^{21}$. Diese Interpretation ist im Großen und Ganzen natürlich richtig und sie zeigt, welch bedeutende Rolle der Krieg in der Politisierung der Nationalsozia-

18 Detlev Peukert, Die Weimarer Republik. Krisenjahre der Klassischen Moderne, Frankfurt a.M. 1987, S. 26-31.

19 Diese beiden Generationen finden sich beispielsweise bei den Kreisleitern der NSDAP in Württemberg: von 64 Kreisleitern wurden 31 zwischen 1891 und 1900 geboren und 25 zwischen 1901 und 1910. Vgl. Christine Arbogast, Herrschaftsinstanzen der württembergischen NSDAP, München 1998, S. 137. Wenn man den Rahmen weiter fasst, wurden von den 1800 von Mathilde Jamin untersuchten SA-Führern 48,5 \% zwischen 1890 und 1901 und 39 \% nach 1902 geboren. Vgl. Mathilde Jamin, Zwischen den Klassen. Zur Sozialstruktur der SA-Führerschaft, Wuppertal 1984, S. 47 und S. 79.

20 Arndt Weinrich, Zwischen Kontinuität und Kritik: Die Hitler-Jugend und die Generation der »Frontkämpfer«. In: Nationalsozialismus und Erster Weltkrieg. Hrsg. von Gerd Krumeich, Essen 2010, S. 271-282, hier S. 273.

21 Nicolas Patin, La guerre au Reichstag: la génération du front entre en politique? In: Parlement[s], 10 (2008), 2, S. 104-117. 
listen gespielt hat. Aber sie vernachlässigt - oder besser gesagt: sie betrachtet nicht genau genug - die Bedeutung des Alters und der generationellen Dynamik für die Ausprägung der politischen Haltung.

Die kommunistische und die nationalsozialistische Partei waren die beiden einzigen Parteien, in denen zwei grundlegende Variablen zusammentrafen, nämlich Kriegserfahrung und Jugend. Diese Feststellung erhärtet die These, dass der »politische Strukturkonflikt der Weimarer Republik wesentlich ein Konflikt der Generationen « gewesen ist ${ }^{22}$. Die traditionellen Parteien wurden letztendlich durch die politischen und sozialen Ereignisse der Jahre 1918/19 nur wenig verändert und blieben damit Vertreterinnen des alten Systems. Nach den Verschiebungen im Parteiensystem Ende 1918, als die alte Deutschkonservative Partei sich mit anderen Gruppierungen zur DNVP zusammenschloss, als es den Liberalen nicht gelang, sich zu vereinigen, und sie so die Trennung zwischen den nationalen und demokratischen Flügeln fortschrieben, blieb als einziges sichtbares Vermächtnis des Krieges die Spaltung der Arbeiterbewegung. Die extremen Parteien konnten also einen großen politischen Gewinn daraus schlagen, dass sie für Bewegung, für Tempo - ja sogar für Gewalt - standen, lauter Eigenschaften, die diesen jungen Menschen die Möglichkeit eines symbolischen und politischen Fortkommens im Kampf gegen die »Herrschaft der Alten $\aleph^{23}$ boten. Die Kommunisten fügten die Gewalt des Krieges in eine bereits gut funktionierende revolutionäre Eschatologie ein, wodurch sie zusätzlich die unbequeme Frage nach der massenhaften Beteiligung ihrer aktiven Mitglieder am »imperialistischen« Krieg umgehen konnten. Die Nationalsozialisten brauchten ihrerseits nur immer wieder lautstark das Erbe des Krieges für sich zu beanspruchen und dafür die Figur des jungen und mutigen Offiziers, des mythischen »Frontkämpfers«, bemühen, der das »System« stürmisch bekämpfte.

Das Argument der Jugend war in einer Partei, in der die meisten ihrer politischen Führer - Hitler, Göring, Röhm - junge Veteranen des Ersten Weltkrieges waren, tatsächlich nicht ungerechtfertigt. Von den 287 Abgeordneten der Nationalsozialisten von Weimar hatten sich 138 zu Beginn des Krieges noch in der Ausbildung befunden ${ }^{24}$. Diese Alterskohorte war natürlich jünger als der Rest der Fraktion, ihr mittleres Geburtsjahr war 1898 (1894 für die gesamte Fraktion). Deshalb gehörten ihr logischerweise auch weniger Veteranen an, denn ein großer Teil von ihr war Teil der für die Front nutzlosen Generation (64 \% Veteranen gegenüber $75 \%$ Gesamtanteil).

Es ist durchaus von Nutzen, sich mit der Karriere der Abgeordneten nach dem Weltkrieg zu befassen. Die Karrieremuster der aktiven Nationalsozialisten sind wohlbekannt: Nach ihrer Entlassung aus dem Militärdienst traten sehr viele von ihnen in Freiwilligenverbände ein. Dort beteiligten sie sich an der Unterdrückung der revolutionären Bewegung in mehr oder weniger radikalen Gruppen wie dem Selbstschutz oder Einwohnerwehren im Reich selbst oder sie kämpften im Grenzschutz. Mit der Auflösung der Freikorps gerieten viele in das völkische Milieu, ein für diese Zeit schwer zu umreißendes Konglomerat rechtsradikaler Gruppie-

Horst Möller, Die Weimarer Republik. Eine unvollendete Demokratie, München 2004, S. 228.

23 Jean Solchany, L'Allemagne au XXe siècle, Paris 2003, S. 103.

24 Hier geht es um alle Männer, die noch zur Volksschule, zur Mittelschule oder zum Gymnasium gingen, eine Lehre machten oder studierten. 
rungen, in dem die NSDAP durchaus keine Vorrangstellung einnahm. Später traten sie dann in drei großen Wellen der nationalsozialistischen Partei bei - zuerst 1920/21, dann 1925 bei der Neugründung der Partei und zuletzt 1929 im Zuge der ersten Wahlerfolge. Für ihre Reichstagsabgeordneten galt im Prinzip das dasselbe Muster, denn 85 von ihnen (30\%) hatten eine Freikorpsvergangenheit, beim Führungskader der SA lag dieser Anteil bei 42 Prozent ${ }^{25}$.

Hier muss man nun genauer hinsehen, denn von diesen 85 Abgeordneten waren nur 67 Veteranen des Weltkrieges. Die anderen 18 (21 \%) hatten nicht im Krieg gedient. Für sie zählte in erster Linie die Ideologie. Ein typisches Beispiel für diese radikale Begeisterung für die Partei ist Hans Frank, ein schwärmerischer junger Student, der sich 1919 an der gewaltsamen Unterdrückung der Münchner Räterepublik beteiligte und in sein Tagebuch schrieb, dass er »für jeden echten Deutschen fürchterlichste Tage und Stunden « durchlebe ${ }^{26}$. Bei ihm wirkte nicht der Krieg als innere Erfahrung, sondern das, was George L. Mosse den »Mythos « genannt hatte - Faszination der Gewalt ja, Brutalisierung durch den Weltkrieg aber keineswegs.

\section{Familientradition}

Krieg ist ein kollektives gesellschaftliches Ereignis, aber manche Menschen bekommen ihn gewissermaßen vererbt. Der Wiener Kongress hatte im 19. Jahrhundert zwar für einen umfassenden Frieden von nie dagewesener Länge in Europa gesorgt, aber Deutschland wurde durch zwei Ereignisse tief geprägt, die Befreiungskriege 1813-1815 und den Deutsch-Französischen Krieg 1870/71. Viele Abgeordnete bezogen sich auf diese historischen Eckdaten, um ihre eigene biografische Entwicklung zu erklären. General a.D. Karl Litzmann etwa, Führer einer Armeegruppe im Weltkrieg und Reichstagsabgeordneter der NSDAP seit November 1932, betonte in seinen Memoiren, dass sein Großvater an den Befreiungskriegen 1813-1815 teilgenommen hatte ${ }^{27}$. Er selbst hatte 1870/71 und 1914-1918 gekämpft. Die polnische Stadt Łódź wurde nach der deutschen Annexion $1940 \mathrm{zu}$ seinen Ehren in Litzmannstadt umbenannt. Die Familiengeschichten und die Weitergabe der Tradition wurden natürlich zur Rechtfertigung vorgetragen, aber für die Akteure waren sie tatsächlich oft Realität.

Ein zweites Beispiel: Friedrich Wilhelm Krüger war 1914 mit zwanzig Jahren Offizier geworden und hatte vier Jahre lang gekämpft. Nachdem die Niederlage besiegelt war, trat er in das Freikorps Lützow ein. 1929 trat er der NSDAP bei und wurde bereits im Juli 1932 Reichstagsabgeordneter. Als er mit dem Gauleiter von Brandenburg und Ostmark, Wilhelm Kube, eine Auseinandersetzung wegen einer Verwaltungsfrage hatte, schrieb er am 13. Oktober 1932: »Persönlich habe ich nicht den Wunsch, Reichstagsabgeordneter zu sein, da ich nicht Politiker, sondern Solkrieg (wie Anm. 20), S. 245.

26 BArch, Nachlass Hans Frank, N 1110/2, Zwei Tagebücher, 1918-1925 (1937, 1942), S. 104: "Es waren das für jeden echten Deutschen fürchterlichste Tage und Stunden.«

27 Karl Litzmann, Lebenserinnerungen, Berlin 1927, Bd 1, S. 5 f. 
dat bin ${ }^{28}$. Z Zwölf Jahre später begründete Krüger in einem Schreiben an Himmler vom 5. März 1944 seinen Antrag auf Versetzung auf einen Posten außerhalb des Generalgouvernements wie folgt: Er habe »das Gefühl und das Bewusstsein, einer Soldatenfamilie abstammend und stets innerlich Soldat gewesen seiend, ein brauchbarer Führer in der Waffen-SS zu werden ${ }^{29}$. Genauso hat Krüger sich selbst gesehen, und zwar bis in die Phase der genozidalen Politik des Dritten Reiches hinein: als Wahrer eines Erbes, das weit über den Ersten Weltkrieg hinausreichte.

Befasst man sich nun aber mit dem Beruf der Väter der 287 nationalsozialistischen Reichstagsabgeordneten und stellt einen Vergleich mit den anderen Parteien an, so lassen sich zwei Punkte deutlich erkennen. Die Aufteilung auf Berufsgruppen ist bei allen Parteien im Großen und Ganzen ähnlich, unabhängig davon, wie man diese Gruppen einteilt ${ }^{30}$. Auffällig ist jedoch, dass Väter in Arbeiterberufen bei den nationalsozialistischen Abgeordneten drastisch unterrepräsentiert waren. Denn während ihr Anteil in den anderen Parteien bei 15 Prozent lag, betrug er bei den Nationalsozialisten nur 6 Prozent. Andererseits hatten nicht einmal 2 Prozent aller Reichstagsabgeordneten einen Soldaten als Vater, während dieser Anteil unter den nationalsozialistischen bei 10 Prozent lag.

Diese Variable betrifft nur eine Gruppe von 19 Abgeordneten, was eine relativ geringe Zahl ist. Aber innerhalb dieser Gruppe findet man idealtypische Karrieren wie die von Erich von den Bach-Zelewski oder Friedrich Wilhelm Krüger, die selbst zumindest ihre ersten beruflichen Erfahrungen als Soldaten sammelten. Sieben der 19 Söhne von Berufssoldaten wurden selbst Berufssoldaten. Wie bei Krüger konnte sich diese Vererbung des Berufs als entscheidend erweisen.

Die Gruppe der ehemaligen Berufssoldaten in der nationalsozialistischen Reichstagsfraktion umfasste 45 Personen (16\%), wobei nicht berücksichtigt ist, ob deren Väter ebenfalls Soldaten waren. Darunter waren die Angehörigen der Generation von 1870, wie Franz Ritter von Epp (1868), Magnus von Levetzow (1871) oder Konstantin Hierl (1875), aber auch jüngere Offiziere wie Ernst Röhm (1887), Franz von Pfeffer (1888), Viktor Lutze (1890) oder Hermann Göring (1893). Logischerweise ist der Veteranenanteil in dieser Gruppe viel höher als im Durchschnitt der restlichen Mitglieder der nationalsozialistischen Fraktion, denn nur zwei Männer waren nicht im Krieg gewesen. Interessanter ist, dass 56 Prozent (25 Abgeordnete) von ihnen einem Freikorps angehört hatten, während das in der gesamten Fraktion nur bei 30 Prozent der Fall war. Im Gegensatz zu den überschwänglichen Studenten, die von der Schulbank direkt in die paramilitärischen Formationen drängten, war dort der Einsatz der Berufssoldaten wohl nicht nur auf ideologische Gründe zurückzuführen. Denn da die Reichswehr durch den Friedensvertrag auf 115000 Mann verkleinert worden war, musste sich ein Großteil der Offiziere beruflich neu orientieren. Die Freikorps oder auch Gliederungen der späteren Schwar-

28 Siehe Nachlass von Friedrich Wilhelm Krüger, BArch, NL 1410/19, Unterlagen als Mitglied des Reichstages, Schreiben vom 13.10.1932: »Persönlich habe ich nicht den Wunsch, Reichstagsabgeordneter zu sein, da ich nicht Politiker, sondern Soldat bin.«

29 BArch, NS 19 2653, S. 102: »sondern das Gefühl und das Bewusstsein, einer Soldatenfamilie abstammend, stets innerlich Soldat gewesen seiend, ein brauchbarer Führer in der Waffen-SS zu werden $\ll$.

30 Martin Döring, »Parlamentarischer Arm der Bewegung«? Die Nationalsozialisten im Reichstag der Weimarer Republik, Düsseldorf 2001, S. 399, zur Diskussion der Berufsgruppen. 
zen Reichswehr ${ }^{31}$ waren damals eine Möglichkeit, eine berufliche Anschlussverwendung zu erlangen. Bevor allerdings weitergehende Schlussfolgerungen gezogen werden können, muss zunächst das Verhältnis von Pragmatismus und Ideologie genauer untersucht werden.

Der Krieg stellte lediglich für einen Bruchteil dieser Gruppe von Soldaten einen beruflichen Einschnitt dar, während bei allen anderen Abgeordneten eine überraschende Kontinuität vorherrschte. Die Studenten setzten ihre akademische Ausbildung fort oder fanden eine Anstellung in ihrem Fachgebiet; die Arbeiter kehrten in ihren Beruf zurück. Für einige von ihnen schien die ländliche Welt - und hier besonders ihr Familienbesitz - ein Rückzugsort zu sein; eine berufliche Option, durch die man mit der kritischen Phase der Demobilmachung und der gesellschaftlichen Neuorientierung in Deutschland 1919/20 und schließlich auch mit der Wirtschaftskrise von 1923 besser fertig werden konnte. Dies gilt beispielsweise für die Reichstagsabgeordneten Werner Willikens und Walter Seidler, die beide während des Krieges Berufssoldaten gewesen waren und anschließend Landwirte wurden. Hier macht sich ein drittes Element bemerkbar, das zum Verständnis der Besonderheit der nationalsozialistischen Fraktion beiträgt: die geografische Herkunft.

\section{Ostgebiete}

In den Familien der nationalsozialistischen Reichstagsabgeordneten war die Landwirtschaft nicht etwa überrepräsentiert. Allerdings sollte man sich hier noch einmal mit den 138 Abgeordneten befassen, die vor dem Krieg noch zur Schule gingen, und sich fragen, welche Ausbildung sie danach verfolgten. Was lernten diese jungen Männer? Vierzig von ihnen gingen an die Universität und wiederum zwanzig von diesen wählten einen Studiengang, der auch rechtswissenschaftliche Lehrinhalte umfasste ${ }^{32}$. Neunzehn machten eine Lehre als Handwerker oder Arbeiter. Aber die größte und kohärenteste Gruppe war die der jungen Männer, die ein landwirtschaftliches Fach erlernten ${ }^{33}$. Acht davon waren selbst Bauernsöhne, was wiederum zeigt, wie wichtig die familiären Netzwerke für die berufliche Eingliederung waren. 70 Prozent hatten am Krieg teilgenommen, und auch hier war, wie bei den Berufssoldaten, der Anteil derjenigen, die in die Freikorps eintraten, mit 40 Prozent größer als im Durchschnitt der NSDAP-Fraktion (30 \%). Diese Gruppe umfasste so unterschiedliche Männer wie Walter Darré, Heinrich Himmler oder Karl Kaufmann, von denen der erste bald der Fachmann der nationalso-

31 Bernhard Sauer, Schwarze Reichswehr und Fememorde. Eine Milieustudie zum Rechtsradikalismus in der Weimarer Republik, Berlin 2004.

32 Hierbei handelt es sich um Studenten, die in einem umfassenden Studienzweig (Geschichte, Philologie, Germanistik, Volkswirtschaft) auch mit der Rechtswissenschaft in Berührung kamen. Dies stimmt weitgehend mit den Schlussfolgerungen von Christian Ingrao über die Bedeutung der Netzwerke an den Universitäten für die Ausbildung der nationalsozialistischen Militanz, vor allem bei den Juristen, überein. Vgl. Ingrao, Hitlers Elite (wie Anm. 9), S. 33-52.

33 Hier habe ich dem Fach Vorrang vor der Institution gegeben. Nur eine sehr kleine Zahl von Männern studierte Landwirtschaft an der Universität. Ich habe sie nicht zu den vierzig Abgeordneten gerechnet, die an der Universität studierten. Die meisten von ihnen studierten in Facheinrichtungen. 
zialistischen Bewegung für landwirtschaftliche Fragen und die Blut-und-BodenIdeologie wurde ${ }^{34}$. Der zweite suchte weiterhin die Nähe zu den Freikorps und studierte zeitgleich Landwirtschaft, weil er sicher war, dort »das Milieu der Reserveoffiziere « $^{35}$ wiederzufinden, das er so schätzte. Der dritte gab seine Vorkriegsstelle als Landwirtschaftshelfer auf und engagierte sich stattdessen fieberhaft in paramilitärischen Verbänden, bevor er sich bereits 1921 der NSDAP anschloss ${ }^{36}$.

Dieser erste, berufsbezogene Ansatz reicht noch nicht aus, um zu verstehen, warum das ländliche Milieu bei den nationalsozialistischen Abgeordneten überrepräsentiert war. Man kann sich also auch mit dem Geburtsort der Abgeordneten befassen. Als Indikator scheint dieser relativ zuverlässig, denn die Deutschen waren damals zwar insgesamt vergleichsweise mobil. Die Jugendlichen wuchsen aber zumeist an ihrem Geburtsort auf, wo ihre Identität ein Stückweit geformt wurde. Bei der Arbeit mit dem Geburtsort gilt es dann nach zwei Kriterien hin zu unterscheiden, nämlich zum einen nach der Herkunft aus städtischen oder ländlichen Gebieten und zum anderen nach der geografischen Herkunft. Die Verteilung der nationalsozialistischen Reichstagsabgeordneten hinsichtlich ihrer geografischen Herkunft entspricht annähernd der Verteilung der damaligen Gesamtbevölkerung, zumindest hinsichtlich des Verhältnisses von ländlichen zu urbanen Gebieten. München und Berlin waren wichtige Zentren, ebenso das Ruhrgebiet und Mitteldeutschland. Zwar wurde ein sehr großer Anteil der NS-Abgeordneten in relativ industrialisierten Gebieten geboren, kam aber tatsächlich aus Dörfern am Rand der städtischen Zentren. Die Abgeordneten aus dem Raum Dresden sind ein gutes Beispiel hierfür, denn nur zwei von ihnen wurden in der Stadt selbst geboren (Hellmuth Körner und Hans Tschammer). Manfred von Killinger hingegen war zwanzig Kilometer weiter westlich gebürtig, aus Gut Lindigt bei Nossen, einer damals weniger als 10000 Einwohner zählenden Kleinstadt. Walter Lingesheim stammte aus Freiberg (30 000 Einwohner), Karl Martin aus Freital (20 000 Einwohner), Herbert Ender aus Borstendorf (unter 2000 Einwohner), Max Wockatz aus Rothnaußlitz (unter 2000 Einwohner) und Robert Helbig aus Bautzen (20 000 Einwohner). Hier lassen sich also zwei Gruppen unterscheiden, von denen die erste aus mittelgroßen Städten und die zweite wirklich vom Land $\mathrm{kam}^{37}$. Dies ist ein wesentlicher Punkt, der aber nicht zu voreiligen Schlüssen führen darf. Die Mythen, denen zufolge sich der Nationalsozialismus im städtischen Arbeitermilieu und in ländlichen Gebieten besonders gut entwickeln konnte, sind von der Forschung längst widerlegt worden. Berlin war unter der Führung von Gauleiter Goebbels ein sehr dynamisches Zentrum des Nationalsozialismus. Benjamin Ziemann hat gezeigt, dass die bayerischen Bauern einer Militarisierung der Veteranen oder einer eventuellen ideologischen Mobilisierung nicht sehr aufgeschlossen gegenüberstanden ${ }^{38}$.

34 Als Beispiel hier eine Veröffentlichung unter Dutzenden anderen: Walther Darré, Das Bauerntum als Lebensquell der nordischen Rasse, München 1940.

35 Peter Longerich, Heinrich Himmler. Biographie, München 2008, S. 34.

36 Karl Höffkes, Hitlers politische Generale. Die Gauleiter des Dritten Reiches, Tübingen 1997, S. 178-182.

37 Ein Vergleich findet sich bei Wolfgang Stelbrink, Die Kreisleiter der NSDAP in Westfalen und Lippe. Versuch einer Kollektivbiographie mit biographischem Anhang, Münster 2003, Tabelle 5, S. 306.

38 Vgl. Benjamin Ziemann, Front und Heimat. Ländliche Kriegserfahrungen im südlichen Bayern, Essen 1997, und Benjamin Ziemann, Das >Fronterlebnis` des Ersten Weltkrieges 
Die Bedeutung der Spaltung zwischen Stadt und Land, die sich auch bei den Wählern zeigte, darf dennoch nicht vernachlässigt werden. Im Demokratisierungsprozess der Republik brachen die alten Risse in der deutschen Gesellschaft wieder auf. Die DNVP gewann schon vor den großen Erfolgen der NSDAP viele Anhänger im ländlichen Bereich, und zwar als Reaktion auf eine Politik, die sich vornehmlich an der städtischen Wählerschaft der SPD orientierte ${ }^{39}$. In dem Moment, wo die Republik Ende der 1920er Jahre in die Krise geriet, wanderte nun ein Großteil dieser Stimmen aus dem »agrarisch-protestantische Milieu « und aus den kleinen Landstädten zur NSDAP40.

Schließlich lässt sich die Herkunft der NSDAP-Abgeordneten auch unter zwei regionalen Aspekten für das gesamte Staatsgebiet betrachten. Einer davon ist relativ bekannt, es handelt sich um den Widerstand der katholischen Gebiete gegen die nationalsozialistische Durchdringung. Das hier untersuchte, überschaubare Sample kann da bestenfalls den durch zahlreiche Untersuchungen inzwischen etablierten Forschungsstand nur noch ergänzen ${ }^{41}$.

Der zweite, bemerkenswerte Punkt ist die große Anzahl der Abgeordneten, die im heutigen Polen (vor allem Posen, Westpreußen, Schlesien) oder sogar noch weiter östlich geboren wurden. 36 Abgeordnete stammten aus den ehemaligen deutschen Ostgebieten. Diese Gebiete waren nun aber alles andere als unbedeutend, denn sie wurden nach den Bestimmungen des Versailler Vertrages bzw. den sich daran anschließenden Volksabstimmungen ganz bzw. teilweise vom Reich abgetrennt und dem neuen Staat Polen zugeschlagen. Für einige dieser Männer befand sich ihre Heimatstadt damit plötzlich im Ausland. Dies galt für Fritz Tiebel, geboren 1889 in Güldenau (Połajewo), oder für Lucian Wysocki, der 1899 in Gentomie (Gętomie) geboren wurde. Erich Ludendorff, Kriegsheld und einflussreicher Erster Generalquartiermeister in der 3. Obersten Heeresleitung von 1916 bis 1918, wurde 1864 in Kruszewnia in der Provinz Posen geboren. In dieser Gruppe von 36 Männern waren 27 Kriegsveteranen (75 \%). Zwölf von ihnen traten in Freikorps ein, darunter drei in das Freikorps, das den Namen einer unmittelbar von der territorialen Umverteilung betroffenen Region trug: »Oberschlesien«. Interessanter ist, dass von den neun Abgeordneten, die nicht am Krieg teilgenommen hatten, aber aus diesen Gebieten stammten, fünf ebenfalls in die Freikorps eintraten. Georg Neugebauer trat schon 1919 in den Grenzschutz von Zduny ein, der etwa hundert Kilometer nördlich seiner Heimatstadt Glatz (Kłodzko) im Einsatz war. Der 1900 in Pyritz (Pyrzyce) geborene Robert Schulz wurde Mitglied des Freikorps Niemeyer, das dem Grenzschutz Ost angehörte. Für diese Männer spielte ihre geografische Herkunft eine entscheidende Rolle. Dies galt auch für den späteren Generalkommissar von Weißruthenien (Weißrussland), Wilhelm Kube, der 1887 im

- eine sozialhistorische Zäsur? Deutungen und Wirkungen in Deutschland und Frankreich. In: Der Erste Weltkrieg und die europäische Nachkriegsordnung. Sozialer Wandel und Formveränderung der Politik. Hrsg. von Hans Mommsen, Köln 2000, S. 43-82. Christian Baechler, L'Allemagne de Weimar 1919-1933, Paris 2007, S. 280.

40 Wolfram Pyta, Politische Kultur und Wahlen in der Weimarer Republik. In: Wahlen und Wahlkämpfe in Deutschland, Von den Anfängen im 19. Jahrhundert bis zur Bundesrepublik. Hrsg. von Gerhard A. Ritter, Düsseldorf 1997, S. 213, und die klassische Untersuchung von Jürgen Falter, Hitlers Wähler, München 1991, vor allem S. 256-266 und S. 154-163.

41 Ian Kershaw, Popular Opinion and Political Dissent in the Third Reich. Bavaria 1933-1945, 2nd rev. ed., Oxford 2002 [Erstausg. 1983]. 
schlesischen Glogau (Głogów) geboren wurde, dort 1918/19 die deutsch-polnischen Auseinandersetzungen und die Teilung der Provinz erlebt hatte und 1928 in seiner ersten Parteifunktion zum Leiter des Gaus Brandenburg ernannt worden war ${ }^{42}$.

\section{Zusammenfassung}

In den Reihen der nationalsozialistischen Reichstagsfraktion befanden sich 75 Prozent Kriegsveteranen: An diesem Ergebnis führt kein Weg vorbei. Der Krieg hatte also auf jeden Fall großen Einfluss auf die Politisierung der Abgeordneten. Dies bestätigt weitgehend die Schlussfolgerungen, die sich aus den Untersuchungen zur »Kriegskultur« ergeben haben.

Aber die Ergebnisse bei den hier untersuchten drei Kriterien - Alter, Beruf des Vaters und geografische Herkunft - machen deutlich, wie wichtig eben auch der biografische Hintergrund war, vor dem die Menschen den Krieg erlebt haben. Selbst innerhalb der relativ kleinen nationalsozialistischen Reichstagsfraktion zeigen sich sehr unterschiedliche Motivationen: Da findet sich der Typ des überschwänglichen Studenten, dem die Kriegsteilnahme verwehrt geblieben war, wie bei Hans Frank, der hauptsächlich ideologisch motiviert wurde. Da findet sich auch der Soldatensohn, der selbst Soldat gewesen war, beispielsweise Friedrich Wilhelm Krüger, der nach dem Krieg in ein Freikorps eintrat. Oder es findet sich ein in den Ostprovinzen des Kaiserreichs geborener Abgeordneter wie Wilhelm Kube, der weder Kriegsteilnehmer noch Mitglied eines Freikorps gewesen war, aber sehr eng mit seiner Geburtsregion verbunden blieb. Die Unterschiede, aber auch die Gemeinsamkeiten zwischen ihnen - vor allem ihre Jugend, der übermäBig hohe Anteil an ehemaligen Berufssoldaten oder die enge Bindung einiger an die Welt der Landwirtschaft - lassen erkennen, dass die Kriegserfahrung nicht in allen gesellschaftlichen Milieus die gleiche Rolle spielte. Niemand wird leugnen, dass der Erste Weltkrieg sowohl ein Trauma als auch einen gesellschaftlichen Katalysator darstellte. Aber dadurch wurden eben nicht mit einem Schlag die Vorprägungen des Kaiserreichs ausgelöscht. Die Worte des Kaisers - »Ich kenne keine Parteien mehr, ich kenne nur noch Deutsche « ${ }^{43}$ - waren natürlich Wunschdenken, denn der Weltkrieg war und blieb stets auch ein innenpolitischer Konflikt ${ }^{44}$. Aus dieser gewählten Perspektive heraus lässt sich also aufzeigen, wie stark doch die Elemente der Kontinuität zwischen Kaiserreich und der Republik waren und dass die Bedeutung der Kriegserfahrungen vielfach nach unten korrigiert werden muss.

Die Männer, die in die NSDAP eintraten, hatten oftmals schon die Erfahrung der Freikorps hinter sich. Wenn man hier aber eine rein kriegerische Dialektik unterstellt, dann vergisst man einerseits, dass auch Männer, die keine Kriegsteilnehmer gewesen waren, in die Freikorps eintraten, und anderseits relativiert man da-

44 Hans-Ulrich Wehler, Deutsche Gesellschaftsgeschichte, Bd 4: Vom Beginn des Ersten Weltkriegs bis zur Gründung der beiden deutschen Staaten, 1914-1949, München 2003, S. 104. 
mit die ideologische, vor allem antikommunistische Prägung dieser Verbände. Es müssen daher zwei Erfahrungsdimensionen deutlich unterschieden werden: einerseits die Erfahrung des Krieges selbst und zum anderen die Erfahrung der Niederlage ${ }^{45}$. Oft war es erst das Erleben der Niederlage, das zu einer dann auch ideologisch motivierten Mobilisierung führte ${ }^{46}$. Die neueren Forschungen zeigen, dass hierfür das bislang verhältnismäßig wenig untersuchte Jahr 1919 entscheidend war ${ }^{47}$.

Die Erforschung der Kriegserfahrung stellt also letztendlich das weit verbreitete Konzept einer »Kriegskultur« wieder in Frage. Erst der Hinweis auf die Unterschiedlichkeit der Kriegserfahrung eröffnet den Blick darauf, wie wenig es historisch gerechtfertigt ist, dass sich die extreme Rechte und später die NSDAP immer wieder als einzige Sachwalter der Frontkämpfer darstellten, obwohl doch der größere Teil der Veteranen sich parteipolitisch eher nach der politischen Mitte oder links orientierte. Erst nachdem die Nationalsozialisten auf politischem Wege an die Macht gelangt waren, konnte sich diese Aneignung ${ }^{48}$ entfalten und konnte sich die NS-Interpretation eines idealen Krieges durchsetzen: Die SA, die um 1930 zu 70 Prozent aus jungen Männern bestand, die nicht im Krieg gewesen waren, konnte nun ohne mit der Wimper zu zucken behaupten, die einzige Erbin dieses kriegerischen Geistes zu sein.

\section{Abstract}

What was the main reason for German people to join the Nazi Party? In the historical literature, the First World War has been often depicted as a major explanation: the conflict is supposed to have created a »war culture « that would have led to the political mobilization of many Nazis. An analysis of the national-socialist members of the Reichstag between 1919 and 1933 does not contradict this hypothesis. Indeed, 80 percent of NSDAP MP's were war veterans. Nevertheless, in other parties too, an enormous proportion of delegates were veterans. Actually other particularities can be identified among Nazi members of the Parliament. The combination of these factors with the war experience can provide a more thorough and realistic picture. Therefore the NSDAP had not the monopoly on war experience, but the one on youth and war experience. The Nazi MP's were ten years younger than the other fractions of the Parliament. Moreover, a higher part of them were career soldiers, and soldiers' sons. They were also more attached to the countryside, by birth, profession, their fathers' professions or the subjects they decided to study. All these criteria lead to question the usual excessive focus on war experience. The Nazi political commitment was much more complex. Nationalsozialismus und Erster Weltkrieg (wie Anm. 20), S. 21-32, hier S. 21 und S. 31.

46 Reichardt, Die SA (wie Anm. 25), S. 245.

47 Das Beispiel des Klischees vom "jüdischen Bolschewismus « wurde von Joachim Schröder entwickelt, der zeigt, dass dieses Klischee durch die mit der Mobilmachung im deutschen Bürgerkrieg verbundene Welle des Antisemitismus im Jahre 1919 verstärkt wurde. Joachim Schröder, Der Erste Weltkrieg und der »jüdische Bolschewismus«. In: Nationalsozialismus und Erster Weltkrieg (wie Anm. 20), S. 77-96, hier S. 94-96. 